\title{
Parameterized Codes over Cycles
}

\author{
Manuel González Sarabia, Joel Nava Lara, Carlos Rentería \\ Márquez and Eliseo Sarmiento Rosales
}

\begin{abstract}
In this paper we will compute the main parameters of the parameterized codes arising from cycles. In the case of odd cycles the corresponding codes are the evaluation codes associated to the projective torus and the results are well known. In the case of even cycles we will compute the length and the dimension of the corresponding codes and also we will find lower and upper bounds for the minimum distance of this kind of codes. In many cases our upper bound is sharper than the Singleton bound.
\end{abstract}

\section{Introduction}

Coding theory is a branch of mathematics that is related to other disciplines, like information theory, computer science, cryptography, graph theory and others. It was born in the middle of the last century with the publication of the work of C.E. Shannon, [14]. In this paper we develop some interesting applications of several tools of commutative algebra and algebraic geometry in order to describe the main parameters of some particular evaluation codes arising from cycles. In fact the bounds for the minimum distance that we find in the subsection 3.2.3 are good enough (for example, our upper bound is sharper than the Singleton bound in many cases). It is worth pointing out that the core of this work is the relation between the parameters of these codes

Key Words: Cycle, Parameterized code, Minimum distance.

2010 Mathematics Subject Classification: Primary 13P25, 14G50; Secondary 14G15, 11T71, 94B27, 94B05.

Received: April, 2013.

Revised: April, 2013.

Accepted: June, 2013. 
(length, dimension, minimum distance) and some invariants of $I_{X}$ (degree, Hilbert function, regularity).

Parameterized codes were introduced in [11]. In that paper the authors computed the length of the parameterized codes associated to any connected simple graph (Corollary 3.8). Unfortunately this is the only parameter that has been found in the general case (for any simple graph). The other two parameters (dimension and minimum distance) must be studied in particular cases. For example, the main parameters of the evaluation codes arising from complete bipartite graphs were described in [5]. We use these approximations in order to study a case that, in spite of the simplicity of the graphs (they are cycles), is very hard.

We start with some necessary definitions.

Let $K=\mathbb{F}_{q}$ be a finite field with $q$ elements and let $Z^{v_{1}}, \ldots, Z^{v_{s}}$ be a finite set of monomials. As usual if $v_{i}=\left(v_{i 1}, \ldots, v_{i n}\right) \in \mathbb{N}^{n}$, then we set

$$
Z^{v_{i}}=Z_{1}^{v_{i 1}} \cdots Z_{n}^{v_{\text {in }}}, \quad i=1, \ldots, s,
$$

where $Z_{1}, \ldots, Z_{n}$ are the indeterminates of a ring of polynomials with coefficients in $K$. Consider the following set parameterized by these monomials

$$
X:=\left\{\left[\left(x_{1}^{v_{11}} \cdots x_{n}^{v_{1 n}}, \ldots, x_{1}^{v_{s 1}} \cdots x_{n}^{v_{s n}}\right)\right] \in \mathbb{P}^{s-1} \mid x_{i} \in K^{*} \text { for all } i\right\},
$$

where $K^{*}=K \backslash\{0\}$ and $\mathbb{P}^{s-1}$ is a projective space over the field $K$. Following [12] we call $X$ an algebraic toric set parameterized by $Z^{v_{1}}, \ldots, Z^{v_{s}}$. The set $X$ is a multiplicative group under componentwise multiplication.

Let $S=K\left[T_{1}, \ldots, T_{s}\right]=\oplus_{d=0}^{\infty} S_{d}$ be a polynomial ring over the field $K$ with the standard grading, let $\left[P_{1}\right], \ldots,\left[P_{|X|}\right]$ be the points of $X$, and let $f_{0}\left(T_{1}, \ldots, T_{s}\right)=T_{1}^{d}$. The evaluation map

$$
\mathrm{ev}_{d}: S_{d}=K\left[T_{1}, \ldots, T_{s}\right]_{d} \rightarrow K^{|X|}, \quad f \mapsto\left(\frac{f\left(P_{1}\right)}{f_{0}\left(P_{1}\right)}, \ldots, \frac{f\left(P_{|X|}\right)}{f_{0}\left(P_{|X|}\right)}\right)
$$

defines a linear map of $K$-vector spaces. The image of $\mathrm{ev}_{d}$, denoted by $C_{X}(d)$, defines a linear code. Following [11] we call $C_{X}(d)$ a parameterized code of order $d$. As usual by a linear code we mean a linear subspace of $K^{|X|}$.

The definition of $C_{X}(d)$ can be extended to any finite subset $X \subset \mathbb{P}^{s-1}$ of a projective space over a field $K$. Indeed if we choose a degree $d \geq 1$, for each $i$ there is $f_{i} \in S_{d}$ such that $f_{i}\left(P_{i}\right) \neq 0$ and we can define $C_{X}(d)$ as the image of the evaluation map given by

$$
\mathrm{ev}_{d}: S_{d}=K\left[T_{1}, \ldots, T_{s}\right]_{d} \rightarrow K^{|X|}, \quad f \mapsto\left(\frac{f\left(P_{1}\right)}{f_{1}\left(P_{1}\right)}, \ldots, \frac{f\left(P_{|X|}\right)}{f_{|X|}\left(P_{|X|}\right)}\right) .
$$


In this generality - the resulting linear code $-C_{X}(d)$ is called an evaluation code associated to $X$ [4]. It is also called a projective Reed-Muller code over the set $X[3,7]$. Some families of evaluation codes have been studied extensively, including several variations of Reed-Muller codes $[2,3,4,5,7,15]$. In this paper we will only deal with parameterized codes over finite fields.

The dimension and length of $C_{X}(d)$ are given by $\operatorname{dim}_{K} C_{X}(d)$ and $|X|$ respectively. The dimension and length are two of the basic parameters of a linear code. A third basic parameter is the minimum distance which is given by

$$
\delta_{X}(d)=\min \left\{\|v\|: 0 \neq v \in C_{X}(d)\right\},
$$

where $\|v\|$ is the number of non-zero entries of $v$. The basic parameters of $C_{X}(d)$ are related by the Singleton bound for the minimum distance

$$
\delta_{X}(d) \leq|X|-\operatorname{dim}_{K} C_{X}(d)+1 .
$$

The parameters of evaluation codes over finite fields have been computed in several cases. If $X=\mathbb{P}^{s-1}$, the parameters of $C_{X}(d)$ are described in [15, Theorem 1]. If $X$ is the image of the affine space $\mathbb{A}^{s-1}$ under the map $\mathbb{A}^{s-1} \rightarrow \mathbb{P}^{s-1}, x \mapsto[(1, x)]$, the parameters of $C_{X}(d)$ are described in $[2$, Theorem 2.6.2]. In this paper we examine the case when $X$ is an algebraic toric set parameterized by $Z_{1} Z_{2}, Z_{2} Z_{3}, \ldots, Z_{n-1} Z_{n}, Z_{n} Z_{1}$.

The vanishing ideal of $X$, denoted by $I_{X}$, is the ideal of $S$ generated by the homogeneous polynomials of $S$ that vanish on $X$.

For all unexplained terminology and additional information we refer to $[1$, 16] (for the theory of polynomial ideals and Hilbert functions), and [10, 17, 18] (for the theory of error-correcting codes and algebraic geometric codes).

\section{Preliminaries}

We continue using the notation and definitions given in the introduction. In this section we introduce the basic algebraic invariants of $S / I_{X}$ and their connection with the basic parameters of parameterized linear codes. Then we present some of the results that will be needed later.

Recall that the projective space of dimension $s-1$ over $K$, denoted by $\mathbb{P}^{s-1}$, is the quotient space

$$
\left(K^{s} \backslash\{0\}\right) / \sim,
$$

where two points $\alpha, \beta$ in $K^{s} \backslash\{0\}$ are equivalent if $\alpha=\lambda \beta$ for some $\lambda \in K$. We denote the equivalence class of $\alpha$ by $[\alpha]$. Let $X \subset \mathbb{P}^{s-1}$ be an algebraic toric set parameterized by $Z^{v_{1}}, \ldots, Z^{v_{s}}$ and let $C_{X}(d)$ be a parameterized code of order $d$. The kernel of the evaluation map ev e $_{d}$, defined in Eq. (1), is precisely 
$I_{X}(d)$ the degree $d$ piece of $I_{X}$. Therefore there is an isomorphism of $K$-vector spaces

$$
S_{d} / I_{X}(d) \simeq C_{X}(d)
$$

Two of the basic parameters of $C_{X}(d)$ can be expressed using Hilbert functions of standard graded algebras [16], as we now explain. Recall that the Hilbert function of $S / I_{X}$ is given by

$$
H_{X}(d):=\operatorname{dim}_{K}\left(S / I_{X}\right)_{d}=\operatorname{dim}_{K} S_{d} / I_{X}(d)=\operatorname{dim}_{K} C_{X}(d) .
$$

The polynomial $h_{X}(t)=\sum_{i=0}^{k-1} c_{i} t^{i} \in \mathbb{Z}[t]$ of degree $k-1=\operatorname{dim}\left(S / I_{X}\right)-$ 1 such that $h_{X}(d)=H_{X}(d)$ for $d \gg 0$ is called the Hilbert polynomial of $S / I_{X}$. The integer $c_{k-1}(k-1)$ !, denoted by $\operatorname{deg}\left(S / I_{X}\right)$, is called the degree or multiplicity of $S / I_{X}$. In our situation $h_{X}(t)$ is a non-zero constant because $S / I_{X}$ has dimension 1. Furthermore $h_{X}(d)=|X|$ for $d \geq|X|-1$, see $[9$, Lecture 13]. This means that $|X|$ equals the degree of $S / I_{X}$. Thus $H_{X}(d)$ and $\operatorname{deg}\left(S / I_{X}\right)$ equal the dimension and the length of $C_{X}(d)$ respectively. There are algebraic methods, based on elimination theory and Gröbner bases, to compute the dimension and the length of $C_{X}(d)$ [11].

The index of regularity of $S / I_{X}$, denoted by $\operatorname{reg}\left(S / I_{X}\right)$, is the least integer $p \geq 0$ such that $h_{X}(d)=H_{X}(d)$ for $d \geq p$. The degree and the regularity index can be read off the Hilbert series as we now explain. The Hilbert series of $S / I_{X}$ can be written as

$$
F_{X}(t):=\sum_{i=0}^{\infty} H_{X}(i) t^{i}=\sum_{i=0}^{\infty} \operatorname{dim}_{K}\left(S / I_{X}\right)_{i} t^{i}=\frac{h_{0}+h_{1} t+\cdots+h_{r} t^{r}}{1-t},
$$

where $h_{0}, \ldots, h_{r}$ are positive integers. Indeed $h_{i}=\operatorname{dim}_{K}\left(S /\left(I_{X}, T_{s}\right)\right)_{i}$ for $0 \leq i \leq r$ and $\operatorname{dim}_{K}\left(S /\left(I_{X}, T_{s}\right)\right)_{i}=0$ for $i>r$. This follows from the fact that $I_{X}$ is a Cohen-Macaulay lattice ideal [11] and by observing that $\left\{T_{s}\right\}$ is a regular system of parameters for $S / I_{X}$ (see [16]). The number $r$ equals the regularity index of $S / I_{X}$ and the degree of $S / I_{X}$ equals $h_{0}+\cdots+h_{r}$ (see [16] or [19, Corollary 4.1.12]).

\section{Parameterized codes arising from cycles}

From now on we will use $s=n$ in the definitions given in the introduction. In our case the number of monomials that parameterize an algebraic toric set is the same that the number of variables in the corresponding polynomial ring. The toric set parameterized by the edges of a cycle $C_{n}$ is given by

$$
X=\left\{\left[\left(a_{1} a_{2}, a_{2} a_{3}, \ldots, a_{n-1} a_{n}, a_{n} a_{1}\right)\right] \in \mathbb{P}^{n-1}: a_{i} \in K^{*} \text { for all } i=1, \ldots, n\right\},
$$


and when we say a projective torus of order $n-1$ we mean the toric set

$$
\mathbb{T}_{n-1}=\left\{\left[\left(a_{1}, \ldots, a_{n}\right)\right] \in \mathbb{P}^{n-1}: a_{i} \in K^{*} \text { for all } i=1, \ldots, n\right\} .
$$

In the next section we will analyze the case where $n$ is an odd number.

\subsection{Odd cycles}

We will take $n=2 k+1$. It is immediate that the set $X$ that appears in the Eq. (3) becomes $\mathbb{T}_{2 k}$ and in this case the parameterized codes $C_{X}(d)$ are called generalized Reed-Solomon codes and their main parameters are well known $([6],[13])$.

Theorem 3.1. The main characteristics of the parameterized codes arising from the edges of an odd cycle $C_{2 k+1}$ are the following:

- Length: $|X|=(q-1)^{2 k}$.

- Vanishing ideal: $I_{X}=\left(\left\{T_{i}^{q-1}-T_{1}^{q-1}\right\}_{i=2}^{2 k+1}\right)$.

- Hilbert series: $F_{X}(t)=\frac{\left(1-t^{q-1}\right)^{2 k}}{(1-t)^{2 k+1}}$.

- Index of regularity: $\operatorname{reg}\left(S / I_{X}\right)=2 k(q-2)$.

- Dimension: $H_{X}(d)=\left(\begin{array}{c}2 k+d \\ d\end{array}\right)+\sum_{i=1}^{2 k}(-1)^{i}\left(\begin{array}{c}2 k \\ i\end{array}\right)\left(\begin{array}{c}2 k+d-i(q-1) \\ d-i(q-1)\end{array}\right)$.

- Minimum distance:

$$
\delta_{X}(d)=\left\{\begin{array}{cl}
(q-1)^{2 k-(m+1)}(q-1-\ell) & \text { if } d \leq 2 k(q-2)-1 \\
1 & \text { if } d \geq 2 k(q-2)
\end{array}\right.
$$

where $m$ and $\ell$ are the unique integers such that $m \geq 0,1 \leq \ell \leq q-2$ and $d=m(q-2)+\ell$.

\subsection{Even cycles}

In this case we will take $n=2 k$. The toric set $X$ is given by Eq. (3). In the following sections we will describe the length and dimension of the parameterized codes arising from even cycles. Finally, we will find lower and upper bounds for the minimum distance of these codes. 


\subsubsection{Length}

In the next theorem we will compute the first parameter of the parameterized codes associated to even cycles.

Theorem 3.2. The length of the parameterized codes arising from the edges of an even cycle $C_{2 k}$ is given by:

$$
|X|=(q-1)^{2 k-2} .
$$

Proof. Let $\varphi$ be the following map:

$$
\begin{aligned}
\varphi: X & \rightarrow \mathbb{T}_{k-1}, \\
\varphi\left(\left[\left(a_{1} a_{2}, a_{2} a_{3}, \ldots, a_{2 k} a_{1}\right)\right]\right) & =\left[\left(a_{1} a_{2}, a_{3} a_{4}, \ldots, a_{2 k-1} a_{2 k}\right)\right] .
\end{aligned}
$$

We note that this map just projects the original vector on its odd components. It is easy to see that $\varphi$ is an homomorphism between the multiplicative groups $X$ and $\mathbb{T}_{k-1}$. In fact if we take $\left[\left(a_{1}, a_{3}, \ldots, a_{2 k-1}\right)\right] \in \mathbb{T}_{k-1}$ we define $b_{2 i}=1$ for all $i=1, \ldots, k$ and $b_{2 i-1}=a_{2 i-1}$ for all $i=1, \ldots, k$. Then the point $\left[\left(b_{1} b_{2}, b_{2} b_{3}, \ldots, b_{2 k} b_{1}\right)\right]=\left[\left(a_{1}, a_{3}, a_{3}, \ldots, a_{2 k-1}, a_{2 k-1}, a_{1}\right)\right] \in X$ and its image under $\varphi$ is $\left[\left(a_{1}, a_{3}, \ldots, a_{2 k-1}\right)\right]$. Therefore $\varphi$ is a surjective map and thus $X / \operatorname{Ker} \varphi$ is isomorphic to $\mathbb{T}_{k-1}$. But

$$
\operatorname{Ker} \varphi=\left\{\left[\left(1, a_{2} a_{3}, 1, a_{4} a_{5}, 1, \ldots, 1, a_{2 k} a_{1}\right)\right]: a_{i} \in K^{*} \text { for all } i\right\},
$$

and then $|\operatorname{Ker} \varphi|=(q-1)^{k-1}$. Therefore $|X|=\left|\mathbb{T}_{k-1}\right| \cdot|\operatorname{Ker} \varphi|=(q-$ $1)^{2 k-2}$.

Remark 3.3. The result given in Eq. (5) is an easy consequence of [11, Corollary 3.8] because even cycles are bipartite graphs. We decided to prove it with the help of the map $\varphi$ because it plays a very important role in the development of this work.

\subsubsection{Dimension}

In this section we will use $S=K\left[T_{1}, \ldots, T_{2 k}\right]$ and $R=K\left[t_{1}, t_{3}, \ldots, t_{2 k-1}\right]$ as the polynomial rings in the corresponding variables. Also $I_{X}$ will be the vanishing ideal of the set $X$ and $I_{\mathbb{T}_{k-1}}$ the corresponding vanishing ideal of the projective torus $\mathbb{T}_{k-1}$. Let $\alpha$ be the map: $\alpha: S \rightarrow R, \alpha\left(T_{2 i-1}\right)=t_{2 i-1}$ for all $i=1, \ldots k, \alpha\left(T_{2 i}\right)=t_{2 i+1}$ for all $i=1, \ldots, k-1$ and $\alpha\left(T_{2 k}\right)=t_{1}$. It means that

$$
\alpha: S \rightarrow R, \alpha\left(\sum_{(i)} a_{(i)} T_{1}^{i_{1}} T_{2}^{i_{2}} \cdots T_{2 k}^{i_{2 k}}\right)=\sum_{(i)} a_{(i)} t_{1}^{i_{1}+i_{2 k}} t_{3}^{i_{2}+i_{3}} \cdots t_{2 k-1}^{i_{2 k-2}+i_{2 k-1}} .
$$


It is immediate that $\alpha$ is a ring epimorphism and in fact we have the next result.

Lemma 3.4. $\alpha\left(I_{X}\right)=I_{\mathbb{T}_{k-1}}$.

Proof. Let $f$ be an homogeneous polynomial in the ring $S$. Then

$$
\alpha(f)=f\left(t_{1}, t_{3}, t_{3}, \ldots, t_{2 k-1}, t_{2 k-1}, t_{1}\right) .
$$

If $\left[\left(a_{1}, a_{3}, \ldots, a_{2 k-1}\right)\right] \in \mathbb{T}_{k-1}$ then, whenever $f \in I_{X}$,

$$
f\left(a_{1}, a_{3}, a_{3}, \ldots, a_{2 k-1}, a_{2 k-1}, a_{1}\right)=0
$$

because $\left[\left(a_{1}, a_{3}, a_{3}, \ldots, a_{2 k-1}, a_{2 k-1}, a_{1}\right)\right] \in X$. Therefore

$$
\alpha(f)\left(a_{1}, a_{3}, \ldots, a_{2 k-1}\right)=f\left(a_{1}, a_{3}, a_{3}, \ldots, a_{2 k-1}, a_{2 k-1}, a_{1}\right)=0 .
$$

Then $\alpha(f) \in I_{\mathbb{T}_{k-1}}$. It proves that $\alpha\left(I_{X}\right) \subseteq I_{\mathbb{T}_{k-1}}$.

By the other hand, we know that (see [6]) $I_{\mathbb{T}_{2 k-1}}=\left(\left\{T_{i}^{q-1}-T_{1}^{q-1}\right\}_{i=2}^{2 k}\right)$ and $I_{\mathbb{T}_{k-1}}=\left(\left\{t_{2 i-1}^{q-1}-t_{1}^{q-1}\right\}_{i=2}^{k}\right)$. Moreover we know that $X \subseteq \mathbb{T}_{2 k-1}$ and it means that $I_{\mathbb{T}_{2 k-1}} \subseteq I_{X}$. Then $t_{2 i-1}^{q-1}-t_{1}^{q-1}=\alpha\left(T_{2 i-1}^{q-1}-T_{1}^{q-1}\right) \in \alpha\left(I_{\mathbb{T}_{2 k-1}}\right) \subseteq \alpha\left(I_{X}\right)$. Therefore $I_{\mathbb{T}_{k-1}} \subseteq \alpha\left(I_{X}\right)$.

From now on we will use the toric set

$$
Y:=\left\{\left[\left(a_{1}, a_{3}, a_{3}, \ldots, a_{2 k-1}, a_{2 k-1}, a_{1}\right)\right] \in \mathbb{T}_{2 k-1}: a_{i} \in K^{*} \text { for all } i\right\} .
$$

Lemma 3.5. The set $Y$ defined in (7) is a complete intersection [3], in fact,

$$
I_{Y}=\left(T_{1}-T_{2 k},\left\{T_{2 i}-T_{2 i+1}\right\}_{i=1}^{k-1},\left\{T_{2 i+1}^{q-1}-T_{2 k}^{q-1}\right\}_{i=1}^{k-1}\right) .
$$

Proof. We will use the lexicographic ordering $T_{1}>T_{2}>\cdots>T_{2 k}$. Let $f \in I_{Y}$. By using the division algorithm, $f$ can be written as

$f=f_{1}\left(T_{1}-T_{2 k}\right)+f_{3}\left(T_{2}-T_{3}\right)+f_{5}\left(T_{4}-T_{5}\right)+\cdots+f_{2 k-1}\left(T_{2 k-2}-T_{2 k-1}\right)+r$,

where $f_{i}, r \in S$ for all $i$, and $r$ is a $K$-linear combination of monomials none of which is divisible by any of $T_{1}, T_{2}, T_{4}, \ldots, T_{2 k-2}$. Then $r\left(T_{1}, \ldots, T_{2 k}\right)=$ $r\left(T_{3}, T_{5}, \ldots, T_{2 k-1}, T_{2 k}\right)$. Let $\left[\left(a_{3}, a_{5}, \ldots, a_{2 k-1}, a_{2 k}\right)\right] \in \mathbb{T}_{k-1}$. Thus

$$
\left[\left(a_{2 k}, a_{3}, a_{3}, \ldots, a_{2 k-1}, a_{2 k-1}, a_{2 k}\right)\right] \in Y
$$

and

$$
0=f\left(a_{2 k}, a_{3}, a_{3}, \ldots, a_{2 k-1}, a_{2 k-1}, a_{2 k}\right)=r\left(a_{3}, a_{5}, \ldots, a_{2 k-1}, a_{2 k}\right) .
$$

Then $r \in I_{\mathbb{T}_{k-1}}$. Therefore (see [6]) $r \in\left(\left\{T_{2 i+1}^{q-1}-T_{2 k}^{q-1}\right\}_{i=1}^{k-1}\right)$ and 


$$
I_{Y} \subseteq\left(T_{1}-T_{2 k},\left\{T_{2 i}-T_{2 i+1}\right\}_{i=1}^{k-1},\left\{T_{2 i+1}^{q-1}-T_{2 k}^{q-1}\right\}_{i=1}^{k-1}\right) .
$$

The inclusion $\supseteq$ is clear and the claim follows.

Lemma 3.6. The vanishing ideal of the toric set $Y$ is given by

$$
I_{Y}=I_{X}+\operatorname{Ker} \alpha .
$$

Proof. The inclusion $I_{Y} \subseteq I_{X}+\operatorname{ker} \alpha$ follows from Lemma 3.5 because $T_{1}-$ $T_{2 k} \in \operatorname{Ker} \alpha,\left\{T_{2 i}-T_{2 i+1}\right\}_{i=1}^{k-1} \subseteq \operatorname{Ker} \alpha$ and $\left\{T_{2 i+1}^{q-1}-T_{2 k}^{q-1}\right\}_{i=1}^{k-1} \subseteq I_{X}$. Let $\left[\left(a_{1}, a_{3}, a_{3}, \ldots, a_{2 k-1}, a_{2 k-1}, a_{1}\right)\right] \in Y$ and $f \in I_{X}, g \in \operatorname{Ker} \alpha$. Then, because $\left[\left(a_{1}, a_{3}, a_{3}, \ldots, a_{2 k-1}, a_{2 k-1}, a_{1}\right)\right] \in X$, we get

$$
f\left(a_{1}, a_{3}, a_{3}, \ldots, a_{2 k-1}, a_{2 k-1}, a_{1}\right)=0 .
$$

Let $g\left(T_{1}, \ldots, T_{2 k}\right)=\sum_{(i)} b_{(i)} T_{1}^{i_{1}} \cdots T_{2 k}^{i_{2 k}}$. Thus

$$
\alpha(g)\left(t_{1}, t_{3}, \ldots, t_{2 k-1}\right)=\sum_{(i)} b_{(i)} t_{1}^{i_{1}+i_{2 k}} t_{3}^{i_{2}+i_{3}} \cdots t_{2 k-1}^{i_{2 k-2}+i_{2 k-1}},
$$

and due to the fact that $\alpha(g)=0$ we conclude that

$$
0=\alpha(g)\left(a_{1}, a_{3}, \ldots, a_{2 k-1}\right)=\sum_{(i)} b_{(i)} a_{1}^{i_{1}+i_{2 k}} a_{3}^{i_{2}+i_{3}} \cdots a_{2 k-1}^{i_{2 k-2}+i_{2 k-1}},
$$

and of course $g\left(a_{1}, a_{3}, a_{3}, \ldots, a_{2 k-1}, a_{2 k-1}, a_{1}\right)=0$.

Therefore $f+g \in I_{Y}$ and the equality (9) follows.

Remark 3.7. We note that $Y \subseteq X \subseteq \mathbb{T}_{2 k-1}$ and then $I_{\mathbb{T}_{2 k-1}} \subseteq I_{X} \subseteq I_{Y}$. Actually we have that

$$
I_{X}+\operatorname{Ker} \alpha=I_{Y}=I_{\mathbb{T}_{2 k-1}}+\operatorname{Ker} \alpha .
$$

In order to compute the dimension of the parameterized codes over an even cycle we need to introduce the map $\bar{\alpha}$.

$$
\bar{\alpha}: S / I_{X} \rightarrow R / I_{\mathbb{T}_{k-1}}, \quad \bar{\alpha}\left(f+I_{X}\right)=\alpha(f)+I_{\mathbb{T}_{k-1}} .
$$

This is a ring epimorphism and in fact we have the following result.

Lemma 3.8. $\operatorname{Ker} \bar{\alpha}=I_{Y} / I_{X}$.

Proof. Let $f+I_{X} \in I_{Y} / I_{X}$. Thus (by Lemma 3.6) $f=g+h$ with $g \in I_{X}$, $h \in \operatorname{Ker} \alpha$. Then $\alpha(f)=\alpha(g)+\alpha(h)=\alpha(g) \in \alpha\left(I_{X}\right)=I_{\mathbb{T}_{k-1}}$ (by Lemma 3.4). Therefore $f+I_{X} \in \operatorname{Ker} \bar{\alpha}$.

By the other hand, let $f+I_{X} \in \operatorname{Ker} \bar{\alpha}$. Then $\alpha(f) \in I_{\mathbb{T}_{k-1}}=\alpha\left(I_{X}\right)$. Then there exists $g \in I_{X}$ so that $\alpha(f)=\alpha(g)$. Thus $f-g \in \operatorname{Ker} \alpha, f \in I_{X}+\operatorname{Ker} \alpha=$ $I_{Y}$ and the result is completely proved. 
Now, we are able to give the main result of this section.

Theorem 3.9. For all $d \geq 0$ the dimension of the parameterized codes arising from the edges of an even cycle $C_{2 k}$ is given by

$$
H_{X}(d)=H_{\bar{\alpha}}(d)+H_{\mathbb{T}_{k-1}}(d),
$$

where $H_{\bar{\alpha}}$ is the Hilbert function of $I_{Y} / I_{X}$, i.e., $H_{\bar{\alpha}}(d)=\operatorname{dim}_{K} I_{Y}(d) / I_{X}(d)$.

Proof. Let $d \geq 0$. Because the map $\bar{\alpha}$ is graded, it induces the surjective linear transformation $\bar{\alpha}(d): S_{d} / I_{X}(d) \rightarrow R_{d} / I_{\mathbb{T}_{k-1}}(d), f+I_{X}(d)=\alpha(f)+$ $I_{\mathbb{T}_{k-1}}(d)$. Then $\operatorname{dim}_{K} S_{d} / I_{X}(d)=\operatorname{dim}_{K} \operatorname{Ker}(\bar{\alpha})(d)+\operatorname{dim}_{K} R_{d} / I_{\mathbb{T}_{k-1}}(d)$. But, by Lemma 3.8, $\operatorname{dim}_{K} \operatorname{Ker}(\bar{\alpha})(d)=\operatorname{dim}_{K} I_{Y}(d) / I_{X}(d)$ and the Eq. (11) follows immediately.

Remark 3.10. We note that

$$
\begin{aligned}
H_{\mathbb{T}_{k-1}}(d)=H_{X}(d)-H_{\bar{\alpha}}(d)= & \operatorname{dim}_{K} S_{d}-\operatorname{dim}_{K} I_{X}(d)-\operatorname{dim}_{K} I_{Y}(d)+ \\
& \operatorname{dim}_{K} I_{X}(d)=\operatorname{dim}_{K} S_{d} / I_{Y}(d)=H_{Y}(d) .
\end{aligned}
$$

In the following corollary and the example given in section 4 , we will use (see Section 2) $r_{X}:=\operatorname{reg}\left(S / I_{X}\right), r_{\mathbb{T}_{k-1}}:=\operatorname{reg}\left(R / I_{\mathbb{T}_{k-1}}\right)$ and $r_{\bar{\alpha}}=$ $\operatorname{reg}\left(I_{Y} / I_{X}\right)$.

Corollary 3.11. With the notation used above, the index of regularity of $S / I_{X}$, where $X$ is the toric set parameterized by the edges of an even cycle $C_{2 k}$, is given by

$$
r_{X}=\max \left\{r_{\bar{\alpha}}, r_{\mathbb{T}_{k-1}}\right\} .
$$

Proof. In order to prove Eq. (12) it suffices to show that the function $H_{\bar{\alpha}}(d)$ is non decreasing (see Eq. (11)). We define the map $\Delta: I_{Y}(d) / I_{X}(d) \rightarrow$ $I_{Y}(d+1) / I_{X}(d+1), \Delta\left(f+I_{X}(d)\right)=T_{1} f+I_{X}(d+1)$. If $f+I_{X}(d) \in \operatorname{Ker} \Delta$ then $T_{1} f \in I_{X}(d+1)$. It means that $\left(T_{1} f\right)\left(a_{1} a_{2}, a_{2} a_{3}, \ldots, a_{2 k} a_{1}\right)=0$ for all $\left[\left(a_{1} a_{2}, a_{2} a_{3}, \ldots, a_{2 k} a_{1}\right)\right] \in X$. Thus

$$
a_{1} a_{2} f\left(a_{1} a_{2}, a_{2} a_{3}, \ldots, a_{2 k} a_{1}\right)=0
$$

but then $f\left(a_{1} a_{2}, a_{2} a_{3}, \ldots, a_{2 k} a_{1}\right)=0$ for all $\left[\left(a_{1} a_{2}, a_{2} a_{3}, \ldots, a_{2 k} a_{1}\right)\right] \in X$ and Ker $\Delta=I_{X}(d)$. Therefore $\operatorname{dim}_{K} I_{Y}(d) / I_{X}(d) \leq \operatorname{dim}_{K} I_{Y}(d+1) / I_{X}(d+1)$ and thus $H_{\bar{\alpha}}(d) \leq H_{\bar{\alpha}}(d+1)$ for all $d \geq 0$. 


\subsubsection{Minimum distance}

In this section we will use the following notation: If $\varphi$ is the map defined in the proof of theorem 3.2 and $M=\operatorname{Ker} \varphi=\left\{\left[R_{1}\right], \ldots,\left[R_{\left|\mathbb{T}_{k-1}\right|}\right]\right\}$ we know that $X=\cup_{i=1}^{\left|\mathbb{T}_{k-1}\right|} M \cdot\left[P_{i}\right]$ (disjoint union of the corresponding cosets) and $|M|=(q-1)^{k-1}$.

In the same way let

$$
\psi: \mathbb{T}_{2 k-1} \rightarrow X, \psi\left(\left[\left(a_{1}, a_{2}, \ldots, a_{2 k}\right)\right]\right)=\left[\left(a_{1} a_{2}, a_{2} a_{3}, \ldots, a_{2 k} a_{1}\right)\right]
$$

The map $\psi$ is a group epimorphism and

$$
\operatorname{Ker}(\psi)=\left\{\left[\left(1, a_{1}, 1, a_{1}, \ldots, 1, a_{1}\right)\right]: a_{1} \in K^{*}\right\}
$$

Therefore if $N:=\operatorname{Ker}(\psi),|N|=q-1$ and $\mathbb{T}_{2 k-1}=\cup_{i=1}^{|X|} N \cdot\left[Q_{i}\right]$ (disjoint union of the corresponding cosets). We will use

$$
N=\left\{\left[U_{1}\right], \ldots,\left[U_{q-1}\right]\right\}
$$

Theorem 3.12. If $\delta_{X}(d)$ is the minimum distance of the parameterized code of order $d$ arising from the edges of an even cycle $C_{2 k}$ then

$$
\frac{\delta_{\mathbb{T}_{2 k-1}}(2 d)}{q-1} \leq \delta_{X}(d) \leq(q-1)^{k-1} \cdot \delta_{\mathbb{T}_{k-1}}(d),
$$

where $\delta_{\mathbb{T}_{2 k-1}}(2 d)$ is the minimum distance of the parameterized code of order $2 d$ associated to the projective torus $\mathbb{T}_{2 k-1}$ and $\delta_{\mathbb{T}_{k-1}}(d)$ is the minimum distance of the parameterized code of order $d$ arising from the projective torus $\mathbb{T}_{k-1}$.

Proof. Let $\Lambda=\left(G\left(Q_{1}\right), \ldots, G\left(Q_{\left|\mathbb{T}_{k-1}\right|}\right)\right) \in C_{\mathbb{T}_{k-1}}(d)$ a codeword with minimum weight where $g\left(t_{1}, t_{3}, \ldots, t_{2 k-1}\right)=\sum_{(i)} a_{(i)} t_{1}^{i_{1}} t_{3}^{i_{3}} \cdots t_{2 k-1}^{i_{2 k-1}} \in R_{d}$ and $G=g / g_{0}$ with $g_{0}\left(t_{1}, t_{3}, \ldots, t_{2 k-1}\right)=t_{1}^{d}$. If $G\left(Q_{i}\right) \neq 0$ we take $M \cdot\left[P_{i}\right]=$ $\varphi^{-1}\left(\left[Q_{i}\right]\right)$. Therefore if $\left[Q_{i}\right]=\left[\left(b_{1}, b_{3}, \ldots, b_{2 k-1}\right)\right]$ then, due to the fact that $\varphi\left(\left[R_{j}\right] \cdot\left[P_{i}\right]\right)=\left[Q_{i}\right]$, we obtain that $\left[R_{j}\right] \cdot\left[P_{i}\right]=\left[\left(b_{1}, *, b_{3}, *, \ldots, b_{2 k-1}, *\right)\right]$. Let $f\left(T_{1}, \ldots, T_{2 k}\right)=\sum_{(i)} a_{i} T_{1}^{i_{1}} T_{3}^{i_{3}} \cdots T_{2 k-1}^{i_{2 k-1}} \in S_{d}$. We will take $F=f / f_{0}$ where $f_{0}\left(T_{1}, \ldots, T_{2 k}\right)=T_{1}^{d}$. Thus $F\left(R_{j} \cdot P_{i}\right)=F\left(b_{1}, *, b_{3}, *, \ldots, b_{2 k-1}, *\right)=$ $G\left(Q_{i}\right) \neq 0$ for all $j=1, \ldots,|M|$. Moreover, if we define the codeword

$$
\Lambda^{\prime}=\left(F\left(R_{1} P_{1}\right), \ldots, F\left(R_{|M|} P_{1}\right), \ldots, F\left(R_{1} P_{\left|\mathbb{T}_{k-1}\right|}\right), \ldots, F\left(R_{|M|} P_{\left|\mathbb{T}_{k-1}\right|}\right)\right)
$$

we have that $\Lambda^{\prime} \in C_{X}(d)$ and if $w\left(\Lambda^{\prime}\right)$ means the Hamming weight of the codeword $\Lambda^{\prime}$ then $w\left(\Lambda^{\prime}\right)=|M| \cdot w(\Lambda)=|M| \cdot \delta_{\mathbb{T}_{k-1}}(d)$ and therefore

$$
\delta_{X}(d) \leq|M| \cdot \delta_{\mathbb{T}_{k-1}}(d)=(q-1)^{k-1} \cdot \delta_{\mathbb{T}_{k-1}}(d)
$$


On the other hand let $\Omega=\left(H\left(P_{1}\right), \ldots, H\left(P_{|X|}\right)\right) \in C_{X}(d)$ a codeword with minimum weight where $h\left(T_{1}, \ldots, T_{2 k}\right)=\sum_{(i)} b_{(i)} T_{1}^{j_{1}} T_{2}^{j_{2}} \cdots T_{2 k}^{j_{2 k}} \in S_{d}$ and $H=h / h_{0}$ where $h_{0}\left(T_{1}, \ldots, T_{2 k}\right)=T_{1}^{d}$. If $H\left(P_{i}\right) \neq 0$ then let $N \cdot\left[Q_{i}\right]=$ $\psi^{-1}\left(\left[P_{i}\right]\right)$ and thus $\psi\left(\left[U_{j}\right] \cdot\left[Q_{i}\right]\right)=\left[P_{i}\right]$ for all $j=1, \ldots, q-1$. Moreover if $\left[P_{i}\right]=\left[\left(a_{1} a_{2}, a_{2} a_{3}, \ldots, a_{2 k} a_{1}\right)\right]$ then we can take $\left[Q_{i}\right]=\left[\left(a_{1}, a_{2}, \ldots, a_{2 k}\right)\right]$ and $\left[U_{j}\right]=\left[\left(1, c_{j}, 1, c_{j}, \ldots, 1, c_{j}\right)\right]$. Let

$$
\ell\left(Z_{1}, \ldots, Z_{2 k}\right)=h\left(Z_{1} Z_{2}, Z_{2} Z_{3}, \ldots, Z_{2 k} Z_{1}\right) \in K\left[Z_{1} Z_{2}, \ldots, Z_{2 k} Z_{1}\right]_{d}
$$

and $L=\ell / \ell_{0}$ where $\ell_{0}\left(Z_{1}, \ldots, Z_{2 k}\right)=\left(Z_{1} Z_{2}\right)^{d}$. Then

$$
\begin{array}{r}
L\left(U_{j} \cdot Q_{i}\right)=L\left(a_{1}, c_{j} a_{2}, a_{3}, c_{j} a_{4}, \ldots, a_{2 k-1}, c_{j} a_{2 k}\right)= \\
H\left(c_{j} a_{1} a_{2}, c_{j} a_{2} a_{3}, \ldots, c_{j} a_{2 k} a_{1}\right)=H\left(a_{1} a_{2}, a_{2} a_{3}, \ldots, a_{2 k} a_{1}\right)=H\left(P_{i}\right) \neq 0
\end{array}
$$

for all $j=1, \ldots, q-1$. Thus if we take the codeword

$$
\Omega^{\prime}=\left(L\left(U_{1} Q_{1}\right), \ldots, L\left(U_{q-1} Q_{1}\right), \ldots, L\left(U_{1} Q_{|X|}\right), \ldots, L\left(U_{q-1} Q_{|X|}\right)\right),
$$

we have that $\Omega^{\prime} \in C_{\mathbb{T}_{2 k-1}}(2 d)$ and $w\left(\Omega^{\prime}\right)=(q-1) \cdot w(\Omega)=(q-1) \cdot \delta_{X}(d)$. Then $\delta_{\mathbb{T}_{2 k-1}}(2 d) \leq(q-1) \cdot \delta_{X}(d)$. Therefore

$$
\delta_{X}(d) \geq \frac{\delta_{\mathbb{T}_{2 k-1}}(2 d)}{q-1},
$$

and the inequalities (14) follow from (15) and (16).

\section{An Example}

We will consider the cycle $C_{6}$ (see Fig. 1) over the finite field $K=\mathbb{Z}_{5}$. In this case the toric set $X$ defined in Eq. (3) becomes

$$
X=\left\{\left[\left(a_{1} a_{2}, a_{2} a_{3}, a_{3} a_{4}, a_{4} a_{5}, a_{5} a_{6}, a_{6} a_{1}\right)\right] \in \mathbb{P}^{5}: a_{i} \in K^{*} \text { for all } i\right\} .
$$

The main parameters of the parameterized code $C_{X}(d)$ arising from the edges of the cycle $C_{6}$ appear below.

- Length: $|X|=(q-1)^{4}=256$.

- Dimension: By using Macaulay2 [8] we obtain the Table 1. There we compute the three different Hilbert functions which appear in Theorem 3.9. Also we note that $r_{X}=r_{\mathbb{T}_{2}}=r_{\bar{\alpha}}=6$. 


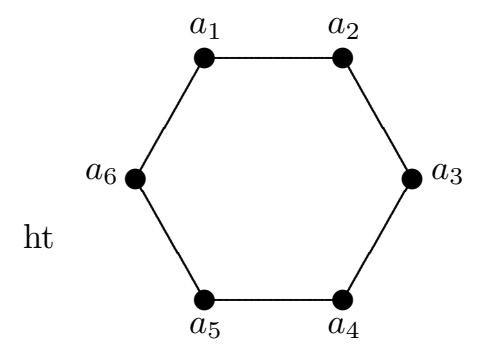

Figure 1: The cycle $C_{6}$

Table 1: Comparison of the different Hilbert fuctions involved in Theorem 3.9.

\begin{tabular}{||c||c||c||c||}
\hline$d$ & $H_{X}(d)$ & $H_{\mathbb{T}_{2}}(d)$ & $H_{\bar{\alpha}}(d)$ \\
\hline 0 & 1 & 1 & 0 \\
\hline 1 & 6 & 3 & 3 \\
\hline 2 & 21 & 6 & 15 \\
\hline 3 & 55 & 10 & 45 \\
\hline 4 & 115 & 13 & 102 \\
\hline 5 & 192 & 15 & 177 \\
\hline 6 & 256 & 16 & 240 \\
\hline
\end{tabular}

Table 2: Bounds for the minimum distance of the parameterized codes arising from the Cycle $C_{6}$.

\begin{tabular}{||c||c||c||c|}
\hline$d$ & $l_{d}$ & $u_{d}$ & $m_{d}$ \\
\hline 1 & 128 & 192 & 249 \\
\hline 2 & 48 & 128 & 236 \\
\hline 3 & 16 & 64 & 202 \\
\hline 4 & 8 & 48 & 142 \\
\hline 5 & 3 & 32 & 65 \\
\hline 6 & 1 & 16 & 1 \\
\hline
\end{tabular}


- Minimum distance: By using the formula to obtain the minimum distance of the parameterized codes arising from the projective torus given in [13, Theorem 3.4] we obtain the Table 2. There we can compare the upper bound for the minimum distance of the parameterized codes over the cycle $C_{6}$ obtained in this work against the Singleton bound. It shows that for $1 \leq d \leq 5$ our upper bound is sharper than the Singleton bound. Also we compute de lower bound for the studied cases. We will use $l_{d}=\frac{\delta_{\mathbb{T}_{5}}(2 d)}{4}$ (lower bound), $u_{d}=(4)^{2} \cdot \delta_{\mathbb{T}_{2}}(d)$ (upper bound) and $m_{d}$ means the Singleton bound.

\section{References}

[1] W. W. Adams and P. Loustaunau, An Introduction to Gröbner Bases (GSM 3, American Mathematical Society, 1994).

[2] P. Delsarte, J. M. Goethals and F. J. MacWilliams, On generalized ReedMuller codes and their relatives, Information and Control 16 (1970) 403442 .

[3] I. M. Duursma, C. Rentería and H. Tapia-Recillas, Reed-Muller codes on complete intersections, Appl. Algebra Engrg. Comm. Comput. 11 (6) (2001) 455-462.

[4] L. Gold, J. Little and H. Schenck, Cayley-Bacharach and evaluation codes on complete intersections, J. Pure Appl. Algebra 196 (1) (2005) 91-99.

[5] M. González-Sarabia and C. Rentería, Evaluation codes associated to complete bipartite graphs, Int. J. Algebra 2 (2008) 163-170.

[6] M. González-Sarabia, C. Rentería and M. Hernández de la Torre, Minimum distance and second generalized Hamming weight of two particular linear codes, Congr. Numer. 161 (2003) 105-116.

[7] M. González-Sarabia, C. Rentería and H. Tapia-Recillas, Reed-Mullertype codes over the Segre variety, Finite Fields Appl. 8 (4) (2002) 511518.

[8] D. Grayson and M. Stillman, Macaulay2 (Available via anonymous ftp from math.uiuc.edu, 1996).

[9] J. Harris, Algebraic Geometry. A first course (Graduate Texts in Mathematics 133, Springer-Verlag, New York, 1992).

[10] F.J. MacWilliams and N.J.A. Sloane, The Theory of Error-correcting Codes (North-Holland, 1977). 
[11] C. Rentería, A. Simis and R. H. Villarreal, Algebraic methods for parameterized codes and invariants of vanishing ideals over finite fields, Finite Fields Appl. 17 (2011) 81-104.

[12] E. Reyes, R. H. Villarreal and L. Zárate, A note on affine toric varieties, Linear Algebra Appl. 318 (2000) 173-179.

[13] E. Sarmiento, M. Vaz Pinto and R. H. Villarreal, The minimum distance of parameterized codes on projective tori, Appl. Algebra Engrg. Comm. Comput. 22 (4) (2011) 249-264.

[14] C.E. Shannon, The mathematical theory of communication, Bell system technical journal 27 (1948) 379-423, 623-656.

[15] A. Sørensen, Projective Reed-Muller codes, IEEE Trans. Inform. Theory 37 (6) (1991) 1567-1576.

[16] R. Stanley, Hilbert functions of graded algebras, Adv. Math. 28 (1978) $57-83$.

[17] H. Stichtenoth, Algebraic function fields and codes (Universitext, Springer-Verlag, Berlin, 1993).

[18] M. Tsfasman, S. Vladut and D. Nogin, Algebraic geometric codes: basic notions (Mathematical Surveys and Monographs 139, American Mathematical Society, Providence, RI, 2007).

[19] R. H. Villarreal, Monomial Algebras (Monographs and Textbooks in Pure and Applied Mathematics 238, Marcel Dekker, New York, 2001).

Manuel GONZÁLEZ SARABIA,

Departamento de Ciencias Básicas,

Unidad Profesional Interdisciplinaria en

Ingeniería y Tecnologías Avanzadas,

Instituto Politécnico Nacional,

07340, México, D.F.

Partially supported by COFAA-IPN and SNI.

Email: mgonzalezsa@ipn.mx

Joel NAVA LARA,

Departamento de Matemáticas,

Escuela Superior de Física y Matemáticas,

Instituto Politécnico Nacional,

07300, México, D.F.

Partially supported by CONACyT.

Email: josiviltj@hotmail.com 
Carlos RENTERÍA MÁRQUEZ,

Departamento de Matemáticas,

Escuela Superior de Física y Matemáticas,

Instituto Politécnico Nacional,

07300, México, D.F.

Partially supported by COFAA-IPN and SNI.

Email: renteri@esfm.ipn.mx

Eliseo SARMIENTO ROSALES,

Departamento de Matemáticas,

Escuela Superior de Física y Matemáticas,

Instituto Politécnico Nacional,

07300, México, D.F.

Partially supported by SNI.

Email: esarmiento@ipn.mx 
